Article

\title{
On Completeness of Sliced Spaces under the Alexandrov Topology
}

\author{
Nazli Kurt ${ }^{1}$ and Kyriakos Papadopoulos ${ }^{2, *(D)}$ \\ 1 Faculty of Science, Open University, P.O. Box 197, Milton Keynes MK7 6BJ, UK; nazkrt.96@gmail.com \\ 2 Department of Mathematics, Kuwait University, P.O. Box 5969, Safat 13060, Kuwait \\ * Correspondence: kyriakos.papadopoulos1981@gmail.com or kyriakos@sci.kuniv.edu.kw
}

Received: 1 December 2019; Accepted: 6 January 2020; Published: 7 January 2020

check for updates

\begin{abstract}
We show that in a sliced spacetime $(V, g)$, global hyperbolicity in $V$ is equivalent to $T_{A}$-completeness of a slice, if and only if the product topology $T_{P}$, on $V$, is equivalent to $T_{A}$, where $T_{A}$ denotes the usual spacetime Alexandrov "interval" topology.
\end{abstract}

Keywords: sliced space; Alexandrov interval topology; global hyperbolicity; slice completeness

\section{Preliminaries}

Sliced spaces have attracted the attention of several authors in studies related to systems of Einstein equations (see [1]), completeness (see [2]), global hyperbolicity (see [3,4]), as well as in problems of a more geometric nature on quantum cosmology (see [5,6]).

Definition 1. Let $V=M \times \mathbb{R}$, where $M$ is an n-dimensional smooth manifold, such that $V$ is equipped with an $n+1$-dimensional Lorentz metric $g$, which splits in the following way:

$$
g=-N^{2}\left(\theta^{0}\right)^{2}+g_{i j} \theta^{i} \theta^{j}
$$

where $\theta^{0}=d t, \theta^{i}=d x^{i}+\beta^{i} d t, N=N\left(t, x^{i}\right)$ is called lapse function, $\beta^{i}\left(t, x^{j}\right)$ is called shift function and $M_{t}=M \times\{t\}$, called spatial slices of $V$, are spacelike submanifolds equipped with the time-dependent spatial metric $g_{t}=g_{i j} d x^{i} d x^{j}$. Such a product space $V$ is called a sliced space.

Let $(V, g)$ be a sliced space. A base for the product topology $T_{P}$, on $V$, consists of all sets of the form $A \times B$, where $A \in T_{M}$ and $B \in T_{\mathbb{R}}$. Here $T_{M}$ denotes the natural topology of the manifold $M$ where, for an appropriate Riemann metric $h$, it has a base consisting of open balls $B_{\epsilon}^{h}(x)$ and $T_{\mathbb{R}}$ is the usual topology on the real line.

The Alexandrov topology (or "interval topology") $T_{A}$ on a spacetime $V$ has a base consisting of open sets of the form $\langle x, y\rangle=I^{+}(x) \cap I^{-}(y)$, where $I^{+}(x)=\{z \in V: x \ll z\}$ and $I^{-}(y)=\{z \in V$ : $z \ll y\}$, where $\ll$ is the chronological order defined as $x \ll y$ iff there exists a future oriented timelike curve, joining $x$ with $y$. By $J^{+}(x)$ one denotes the topological closure of $I^{+}(x)$ and by $J^{-}(y)$ that one of $I^{-}(y)$ (see [7]).

A spacetime $V$ is strongly causal, if and only if it is strongly causal at every point, that is, for every point $p \in V, p$ has arbitrarily small causally convex neighbourhoods. We say that $V$ is globally hyperbolic, if and only if $V$ is strongly causal and every set $J^{+}(x) \cap J^{-}(y)$ (called a "closed diamond") is compact. Global hyperbolicity is considered the strongest causality condition in the causal hierarchy of spacetimes (see [8]) and is equivalent to the existence of a Cauchy hypersurface $S$ for $V$ (see Section 5, in [7]); this supplies us with the benefit to construct on $V$ well-defined initial-value problems (see $[9,10]$, Theorem 10.2.2). One can also view global hyperbolicity as a property on a spacetime 
which guarantees the absence of naked singularities in $V$ (for its role in the strong cosmic censorship, see [11]).

In the next section we will show that global hyperbolicity in a sliced spacetime $(V, g)$ is equivalent to completeness with respect to the Alexandrov topology of a slice $\left(M_{t}, g_{t}\right)$. Although completeness of the Alexandrov topology $T_{A}$, by itself, is not a criterion of nonsingularity (in the Schwartzschild space and the Friedmann-Robertson-Walker cosmologies, for example, $T_{A}$ is complete, but these spaces are singular; see [12]), it is interesting that in the particular case of sliced spacetimes that are equipped with their natural product topology, completeness of a slice with respect to $T_{A}$ can be considered as a criterion of global hyperbolicity for the entire space.

Thoughout our text, for topological terms like Hausdorff space and completeness, we refer to the seminal book of Engelking, [13].

\section{A Topological Condition for the Completeness of a Sliced Space}

In [3], sliced spaces are being considered to have uniformly bounded lapse, shift and spatial metric, in order to achieve the equivalence of global hyperbolicity of $(V, g)$ with the completeness of the slice $\left(M_{0}, \gamma\right)$ (Theorem 2.1). Being motivated by this result, in the Theorem that follows, we consider global topological conditions, for showing the equivalence of global hyperbolicity of $(V, g)$ with a slice $\left(M_{t}, g_{t}\right)$ being $T_{A}$-complete. Our Theorem 1, below, differs from Theorem 2.1 of [3] in that the slices in [3] are complete Riemannian manifolds (with uniformly bounded spatial metric, lapse and shift functions) while in our case the slices are $T_{A}$-complete. We discuss this further in Section 3.

Theorem 1. Let $(V, g)$ be a sliced space, with respect to its natural product topology $T_{P}$, where $V=M \times \mathbb{R}$, $M$ is an $n$-dimensional manifold and $g$ the $n+1$-Lorentz "metric" on $V$. Let also $T_{A}$ be the Alexandrov topology on $V$. Then, the following statements are equivalent:

1. $(V, g)$ is globally hyperbolic.

2. $T_{P} \equiv T_{A}$.

3. $\left(M_{t}, g_{t}\right)$ is complete with respect to $T_{A}$.

Proof. 1. has been shown to be equivalent to 2. in [4].

To show that 2. implies 3., we first notice that since $(V, g)$ is globally hyperbolic, it is also strongly causal. Since, also, $T_{P} \equiv T_{A}$, we have that for every $t \in \mathbb{R}, M_{t}$ is a subset of a spacetime $V$, with nondegenerate spacetime metric, with subspace topology $T_{A}$ inherited from $V$, such that $M_{t}$ is strongly causal. Hence, $T_{A}$, on $M_{t}$, is complete (see [12], Theorem 2).

For proving that 3. implies 1., for each $t \in \mathbb{R}$, we let $\left(M_{t}, g_{t}\right)$ to be complete with respect to $T_{A}$, where each $M_{t}$ is a spacelike submanifold with time dependent spatial metric $g_{t} \equiv g_{i j} d x^{i} d x^{j}$. But since each $M_{t}$ is complete, the Alexandrov topology $T_{A}$, on $M_{t}$, is strongly causal (see, again, [12]). So, each point of $M_{t}$ is strongly causal, which means that for every point $P \in M_{t}$ there exists an arbitrarily small convex neighbourhood. But, $V=\bigcup_{t \in \mathbb{R}} M \times\{t\}$, so $P \in V$ if and only if there exists $M_{t}=M \times\{t\}$, such that $P \in M_{t}$, and hence $V$ is strongly causal with respect to $T_{A}$. That the closed $T_{A}$-diamonds, in $V$, are compact, has been shown in Theorem 3, from [4]. Thus, $(V, g)$ is globally hyperbolic.

\section{Discussion}

Question 1: Can our Theorem 1 hold, if one substituted in 3. " $\left(M_{t}, g_{t}\right)$ is complete with respect to $T_{A}$ " with the statement " $\left(M_{t}, g_{t}\right)$ is a complete Riemannian manifold"? The answer is negative, since in a spacetime manifold, $T_{A}$ is usually a coarser topology than the spacetime topology, and it is equivalent to the manifold topology only if it is Hausdorff (see [7], Theorem 4.24). So, in order for this question to have a positive answer, one would have to add in Theorem 1 the extra condition that $T_{A}$, on $V$, is Hausdorff. As a continuation of this question, we ask whether the spacetimes considered in [3] may well have their Alexandrov topology $T_{A}$ not being Hausdorff. In such a case, strong causality will fail due to this (see, for example, Remark 4.25 of [7]). Spacetimes where $T_{A}$ fails to be Hausdorff, 
according to Penrose, admit a null geodesic along which strong causality fails and this is one aspect of a general result concerning the region of strong causality failure in a spacetime [7]. Given the above argument, we conjecture that for a physically reasonable spacetime, the statements of Theorem 2.1 of [3] and of Theorem 1 here should be equivalent. A rigorous proof showing the equivalence of a uniformly bounded spatial metric, lapse and shift functions with the condition of the topologies $T_{P}$ and $T_{A}$ to be equivalent, will be of a great interest.

In [3], there are conditions introduced, so that global hyperbolicity to be equivalent to geodesic completeness. In particular, in Theorem 3.1, the term trivially sliced space is introduced, so that a slice is a complete Riemannian manifold, if and only if the space $(V, g)$ is geodesically complete. The "disadvantage" of this condition is that the spatial metric $g_{i j}$ is time-independent.

Question 2: Can one relate slice-completeness and geodesic completeness of $(V, g)$ with a time-dependent spatial metric $g_{i j}$ ?

Question 2 does not seem to have a trivial answer. In a possible variation of Theorem 1, towards an answer to this question, one could make use of the classical Hopf-Rinow Theorem (see [14]), which gives that metric completeness, in a spacetime, is equivalent to geodesic completeness. Again, $T_{A}$ should be Hausdorff.

Author Contributions: Conceptualization, N.K. and K.P.; methodology, N.K. and K.P.; investigation, N.K. and K.P.; resources, N.K. and K.P.; writing-original draft preparation, K.P.; writing-review and editing, K.P.; supervision, K.P., project administration, N.K. and K.P. The both authors have read and agreed to the published version of the manuscript.

Funding: This research received no external funding.

Acknowledgments: The authors would like to thank Albert Much, for the oral communication and for the motivation, which led to this note. In particular, Question 2 is credited to him. Many thanks to the two unknown referees for their remarks and additions, which improved the shape of the text significantly.

Conflicts of Interest: The authors declare no conflicts of interest.

\section{References}

1. Choquet-Bruhat, Y.; Ruggeri, T. Hyperbolicity of the $3+1$ system of Einstein equations. Commun. Math. Phys. 1983, 89, 269-275. [CrossRef]

2. Choquet-Bruhat, Y.; Cotsakis, S. Global Hyperbolicity and Completeness. J. Geom. Phys. 2002, 43, 345-350. [CrossRef]

3. Cotsakis, S. Global Hyperbolicity of Sliced Spaces. Gen. Relativ. Gravit. 2004, 36, 1183-1188. [CrossRef]

4. Papadopoulos, K.; Kurt, N.; Papadopoulos, B.K. On Sliced Spaces; Global Hyperbolicity Revisited. Symmetry 2019, 11, 304. [CrossRef]

5. Finster, F.; Much, A.; Oeckl, R. Stationary Spacetimes and Self-Adjointness in Klein-Gordon Theory. arXiv 2020, arXiv:1910.05322.

6. Much, A.; Oeckl, R. Self-Adjointness in Klein-Gordon Theory on Globally Hyperbolic Spacetimes. arXiv 2018, arXiv:1804.07782.

7. Penrose, R. Techniques of Differential Topology in Relativity. In CBMS-NSF Regional Conference Series in Applied Mathematics; 1972. Available Online: https://www.amazon.com/Techniques-Differential-RelativityConference-Mathematics/dp/0898710057 (accessed on 7 December 2019).

8. Minguzzi, E. Lorentzian causality theory. Living Rev. Relativ. 2019, 22, 3. [CrossRef]

9. Choquet-Bruhat, Y.; Geroch, R. Global aspects of the Cauchy problem in general relativity. Commun. Math. Phys. 1969, 14, 329-335. [CrossRef]

10. Wald, R.M. General Relativity; The University of Chicago Press: Chicago, IL, USA, 1984.

11. Penrose, R. Gravitational collapse-The role of general relativity. Gen. Relativ. Gravit. 2002, 34, 1142-1164. [CrossRef] 
12. McWilliams, K.J. Completeness of the Alexandrov topology for space-time. Int. J. Theor. Phys. 1981, $20,905$. [CrossRef]

13. Engelking, R. General Topology; Sigma Series in Pure Mathematics; Heldermann Verlag: Berlin, Germany, 1989; Volume 6, ISBN 3-88538-006-4. Available Online: http:/ / www.heldermann.de/SSPM/SSPM06/sspm06.htm (accessed on 7 December 2019).

14. Hopf, H.; Rinow, W. Ueber den Begriff der vollständigen differentialgeometrischen Fläche. Comment. Math. Helv. 1931, 3, 209-225. [CrossRef]

(C) 2020 by the authors. Licensee MDPI, Basel, Switzerland. This article is an open access article distributed under the terms and conditions of the Creative Commons Attribution (CC BY) license (http://creativecommons.org/licenses/by/4.0/). 